\section{The fallacy of the cross-modal Stroop effect: A rejoinder to Cowan (1989)}

\section{CHRISTOPHER MILES and DYLAN M. JONES University of Wales College of Cardiff Cardiff, Wales}

The aims of this short rejoinder to Cowan (1989) are twofold. First, we provide data further supporting the position of Miles, Madden, and Jones (1989). Second, we question the theoretical validity of equating the "phonological store" (see Miles et al., 1989) with the "prespeech buffer"' (see Cowan \& Barron, 1987).

\section{An Attempt at Further Replication of Cowan and Barron (1987)}

Cowan (1989) pointed to a number of methodological differences between Cowan and Barron's (1987) study and the attempted replications reported by Miles et al. (1989). In particular, the studies differed in (1) the rate of presentation of auditory stimuli (1.66 words/sec and $1 \mathrm{word} / \mathrm{sec}$, respectively), and (2) the size of the potential response set (five items and four items, respectively). It is acknowledged that these methodological differences may plausibly account for the observed discrepancy in the data.

Because of this possibility, the experiment reported here adopted a procedure identical in all respects to that of Cowan and Barron (1987), with the exception that the 30 subjects (19 women and 11 men) completed the task in only two auditory conditions: silence and spoken color words. Auditory material (a male voice) was presented in digitized form over headphones at a rate of 1.6 words/sec and at an intensity of $75 \mathrm{dBA}$. This intensity level is some $10 \mathrm{dBA}$ below Cowan and Barron's reported intensity of $85 \mathrm{dBA}$ (see Cowan, 1989, Note 2). However, as Cowan argued, if the comparison is with silence, then intensity is unimportant. For details of the visual materials, see Cowan and Barron (1987).

Both reading times and error rates were recorded. A $2 \times 2$ analysis of variance (ANOVA) was performed on the reading times with visual condition (color words and $\mathrm{Xs}$ ) and auditory condition (spoken color words and silence) as within-subjects factors. The main effect of visual materials $[F(1,29)=496.07, p<.001]$ reflected lengthened reading times for the color-word (CW) card. However, neither the main effect of auditory condition $[F(1,29)=0.21]$ nor the interaction effect $[F(1,29)=$ $0.41]$ approached significance (see Table 1). The lengthened reading times with spoken color words, predicted

\footnotetext{
This work was funded by a grant from the United Kingdom's Economic and Social Research Council. Neil Morris provided useful discussion in the preparation of this reply. Address correspondence to Christopher Miles, School of Psychology, University of Wales College of Cardiff, P.O. Box 901, Cardiff CF1 3YG, U.K.
}

by Cowan (1989), occurred in only 10 subjects, with 20 producing reading times in the opposite direction ( $p>.89$, sign test). With respect to reading errors, a $2 \times 2$ ANOVA revealed a main effect of visual materials $[F(1,29)=37.33, p<.001]$. Again, neither the main effect of auditory condition $[F(1,29)=0.22]$ nor the interaction effect approached significance $[F(1,29)=0.09$; see Table 1].

Clearly, these data unequivocally failed to confirm the results presented by Cowan and Barron (1987). Reading times in this study for the spoken color-word condition are comparable across visual materials to those reported by Cowan and Barron; however, the error rate reported here is below that reported by Cowan and Barron (1987; see Figure 2).

In the present study, the subjects encountered spoken color-word interference on two out of four trials (50\%), compared with 2 out of 10 trials (20\%) in Cowan and Barron. However, in absolute terms, subjects in both studies received the same number of spoken color-word trials. Therefore, Cowan's (1989) suggestion that the crossmodal Stroop interference effect might attenuate with increased experience cannot account for our failure to replicate Cowan and Barron's original findings.

In sum, the present study, with only minor procedural differences from Cowan and Barron's study, produced data completely consistent with our earlier findings, and in direct contradiction to the data of those authors.

\section{Theoretical Considerations}

Both parties agree that irrelevant speech does not affect perception of visual stimuli per se, but exerts its effect later in the processing system. Cowan (1989) argued that spoken color words have privileged access to a prespeech buffer, and therein compete for output with visually presented color words (or colors) that are to be named. Crucial to his argument is the notion that it is the words, or their appropriate codes (whether visually or auditorily presented), that compete for output. In contrast, Miles et al. (1989), in line with Salamé and Baddeley (1982), suggested that the effect of irrelevant speech works in a rather different way. They predicted that effects of irrelevant speech should occur only on tasks involving short-term memory, and that interference is due to confusion of phonemic codes (see Miles et al., 1988, 1989 , for a full discussion).

Table 1

Mean Reading Times (in sec) and Percent Error Rates (\%E) for Auditory and Visual Conditions

\begin{tabular}{|c|c|c|c|c|}
\hline \multirow{3}{*}{$\begin{array}{c}\text { Auditory } \\
\text { Condition }\end{array}$} & \multicolumn{4}{|c|}{ Visual Condition } \\
\hline & \multicolumn{2}{|c|}{ Color Words } & \multicolumn{2}{|c|}{$\mathrm{Xs}$} \\
\hline & $\boldsymbol{M}$ & $\% \mathrm{E}$ & $M$ & $\% \mathrm{E}$ \\
\hline Spoken Color Words & 83.89 & 1.23 & 65.62 & 0.16 \\
\hline Silence & 84.86 & 1.33 & 65.58 & 0.2 \\
\hline
\end{tabular}


We argue that the assumed properties of the prespeech buffer and phonemic store differ in several important respects. Such differences render untenable Cowan's (1989) suggestion that Cowan and Barron's (1987) data can be interpreted with reference to the phonemic store.

First, the phonemic store is utilized in short-term memory tasks to help retain the fidelity of to-beremembered items during the rehearsal period. Because the store has a limited capacity, it is particularly susceptible to disruption by irrelevant speech when the memory load is high. With smaller memory loads, the store is not prone to such disruption. Indeed, we have shown that the Stroop task, with a single-item memory load, is insensitive to the effects of irrelevant speech. Therefore, we would predict effects of auditory speech material on memory tasks (via the phonemic store), but not on the Stroop task.

Second, effects of irrelevant speech are observed on immediate memory tasks without the necessity of a spoken response (see Jones, Miles, \& Page, in press). Critical to Cowan's theoretical position is the necessity for spoken output (see Cowan, 1989).

Third, the phonemic store is sensitive to auditory material that represents a different category from that material presented visually (e.g., spoken words disrupt memory for digits). Cowan suggested a much more specific effect, which would be observed only when the visual and auditory information represent the same category (in this instance, color words). Indeed, were the phonemic store utilized in the cross-modal Stroop phenomenon, as Cowan suggested is possible, this would predict an effect of repetitions of the alphabet in Cowan and Barron's (1987) study. Clearly, the spoken alphabet consists of phonemic material.

In conclusion, we have presented data further questioning the reliability of Cowan and Barron's (1987) study. In addition, a number of theoretical issues are outlined which suggest that it is theoretically untenable to equate the prespeech buffer with the phonemic store.

\section{REFERENCES}

Cowan, N. (1989). A reply to Miles, Madden, and Jones (1989): Mistakes and other flaws in the challenge to the cross-modal Stroop effect. Perception \& Psychophysics, 45, 82-84.

Cowan, N., \& Barron, A. (1987). Cross-modal, auditory-visual Stroop interference and possible implications for speech memory. Perception \& Psychophysics, 41, 393-401.

Jones, D. M., Miles, C., PAGE, J. (in press). Disruption of proofreading by irrelevant speech: Effects of attention, arousal or memory? Applied Cognitive Psychology.

Miles, C., Madden, C., \&ones, D. M. (1989). Cross-modal, auditory-visual Stroop interference: A reply to Cowan and Barron (1987). Perception \& Psychophysics, 45, 77-81.

Miles, C., Madden, C. \& Jones, D. M. (1988). Levels of interference by irrelevant speech: One, some or many? In A. Colley \& J. Beech (Eds.), Cognition and action in skilled behaviour (pp. 349-359). Amsterdam: Elsevier Science Publishers, North-Holland.

Salamé, P., \& Baddeley, A. D. (1982). Distuption of short-term memory by unattended speech: Implications for the structure of working memory. Journal of Verbal Learning \& Verbal Behavior, 21. 150-164.

(Revision accepted for publication June 10, 1988.) 